\title{
STRUCTURAL AND MAGNETIC CHARACTERIZATION OF POLYANILINE COMPOSITE FILMS
}

\author{
J. C. APHESTEGUY ${ }^{1}, \underline{\text { S.E. JACOBO }}{ }^{1}$, R. LÓPEZ ANTÓN ${ }^{2}$, G.V. KURLYANDSKAYA ${ }^{2}$ \\ ${ }^{1}$ LAFMACEL, Facultad de Ingeniería, Univ. Nac. de Buenos Aires, Buenos Aires, Argentina. E- \\ mail: \\ ${ }^{2}$ Universidad del País Vasco UPV-EHU, Depto Electricidad y Electrónica, Bilbao, Spain \\ caphestegu@fi.uba.ar, sjacobo@fi.uba.ar,galina@uniovi.es
}

Keywords: polyaniline (PANI), composites, electrical and magnetic properties, nanoparticles

\begin{abstract}
In this work a novel approach for the preparation of $\mathrm{Fe}_{3} \mathrm{O}_{4} / \mathrm{PANI}$ (polyaniline) thin film composite containing magnetic nanoparticles is presented. Magnetite $\left(\mathrm{Fe}_{3} \mathrm{O}_{4}\right)$ nanoparticles have been coated by PANI and the PANI chains have been doped by 10-camphorsulfonic acid (CSA). The doped composite is soluble in common organic solvents. Thin films of composites of polyaniline (PANI) were casted from m-cresol. Several characterization techniques were employed in order to determine composition, structure and magnetic properties of the nanocomposite film (Xray diffraction, transmission electron microscopy, TEM, Scanning electron microscopy, SEM, and optical microscopy). The magnetization data were obtained from $\mathrm{M}(\mathrm{H})$ hysteresis loops and zero field cooling - field cooling, ZFC-FC. Magnetic measurements evidence a ferromagnetic behaviour of the obtained composite, at room temperature with saturation magnetization of about $3.4 \mathrm{emu} / \mathrm{g}$ and coercivity of 42 Oe.

The temperature dependences of the conductivity of the films follows the $\sigma(T)=\sigma_{o} \exp \left[-\left(T_{o} / T\right)^{1 / 2}\right]$ law, which has been explained within the framework of the onedimensional variable-range-hoping (1D-VRH) model. Application of 1T magnetic field increases the resistivity of the film and the temperature slope dependence.
\end{abstract}

\section{Introduction.}

Magnetic nanoparticles attract broad interest in different areas such as high density data storage [13], color imaging, sensors, biomedical applications [4], and spintronics [5]. Nanoparticles show unusual phenomena comparing with bulk or microscale size magnets of the same composition and diverse areas of their applications directly depend on the level of the understanding of the properties.

Ultrafine particles embedded in a metallic, polymer, etc. matrix or spread on the surface is often called nanoclusters. It is desirable to endow the magnetic nanoclusters with highly controlled reproducible processing. Nanocomposites with high electrical conductivity demand both very careful design and accurate characterization. For their preparation the conducting polymer matrixes must suit the condition to be soluble and stable. Several methods have been employed to prepare magnetic nanoclusters embedded in conducting polymers in order to obtain composites possessing the unique magnetic, electrical, and optical properties. Wan and coworkers prepared magnetite/PANI nanocomposites by mixing aqueous solutions of iron (II) sulfate and 1-methyl-2pyrrolidinone (NMP) solutions of PANI at different $\mathrm{pH}$ values [6]. B.Z. Tang et al developed a versatile process for the preparation of $\mathrm{Y}-\mathrm{Fe}_{2} \mathrm{O}_{3} / \mathrm{PANI}$ nanocomposite films with high electrical conductivity and magnetical susceptibility [7].

In this work we present a novel approach for the preparation of $\mathrm{Fe}_{3} \mathrm{O}_{4} / \mathrm{PANI}$ (polyaniline) thin film composite containing magnetic nanoparticles. Magnetic and transport properties are discussed. 


\section{Experimental.}

$\mathrm{Fe}_{3} \mathrm{O}_{4} / \mathrm{PANI}$ nanoparticles were prepared "in situ" as reported earlier [8] from a mixed aqueous solution of $\mathrm{FeCl}_{2} \cdot 4 \mathrm{H}_{2} \mathrm{O}(1.0 \mathrm{M})$ and $\mathrm{FeCl}_{3} \cdot 6 \mathrm{H}_{2} \mathrm{O}(1.5 \mathrm{M})$ as oxidant and a specific volume of the aniline $(\mathrm{PhN})$ in chloride solution. The resulting composite was precipitate with the addition of a concentrated ammonium solution. The $\mathrm{pH}$ values of the reaction mixture were kept in the range 910. The resulting nanoparticles $\mathrm{Fe}_{3} \mathrm{O}_{4}$ :PANI (1:0.2) were filtered, washed with distilled water and dried under vacuum at $40^{\circ} \mathrm{C}$ for $24 \mathrm{hrs}$. Previously, PANI power, in the base form, was prepared through an oxidative route as everyboby knows [8-9]. Appropiate amounts of dried powders of pure PANI and $\mathrm{Fe}_{3} \mathrm{O}_{4} / \mathrm{PANI}$ were weighted and mixed and ground carefully. In this way we obtained a diluted $\mathrm{Fe}_{3} \mathrm{O}_{4} / \mathrm{PANI}$ nanocomposite where $\mathrm{Fe}_{3} \mathrm{O}_{4}(8 \% \mathrm{w} / \mathrm{w}$ determined by termogravimetric analysis) is the magnetic core, and PANI the conducting shell. The ferromagnetic composite was then doped with camphorsulfonic acid, $\mathrm{CSA}$, in the molar ratio $\mathrm{H}^{+} / \mathrm{PhN}=0.5$, which increases conductivity and solubility in organic solvents as $m$-cresol [7]. The composite thin films of the thickness of $30 \mu \mathrm{m}$ were prepared by simple static casting of the composite m-cresol solution on a glass substrate having $1.0 \times 0.5 \mathrm{~cm}^{2}$. As-prepared by casting thin films were vacuum dried overnight at $50^{\circ} \mathrm{C}$.

The surface morphology of the cast films was studied by optical microscopy using a Nikon LUEPI microscope with digital camera. The morphologies of films and particles were measured by optical microscopy (Nikon L-UEPI microscope with digital camera) scanning electron microscope, SEM (JEOL JSM 6400), and a transmission electron miscroscope, TEM (JEM 200).

Magnetic properties of the films were measured at room and low temperatures using a SQUID magnetometer (Quantum Design MPMS). For SQUID measurements the composite thin film sample of about $0.60 \mathrm{mg}$ (approximately $4 \times 4 \mathrm{~mm}^{2}$ ) was separated from the glass substrate. The temperature dependence of conductivity was performed in a Physical Properties Measurement System (PPMS from Quantum Design). Following the four-in-line geometry, copper wires were attached by silver paint for the measurement of sample resistance. Transport measures of films were performed too, in the presence of a magnetic field (1T) perpendicular to the film surface and current direction.

\section{Results and Discussion}

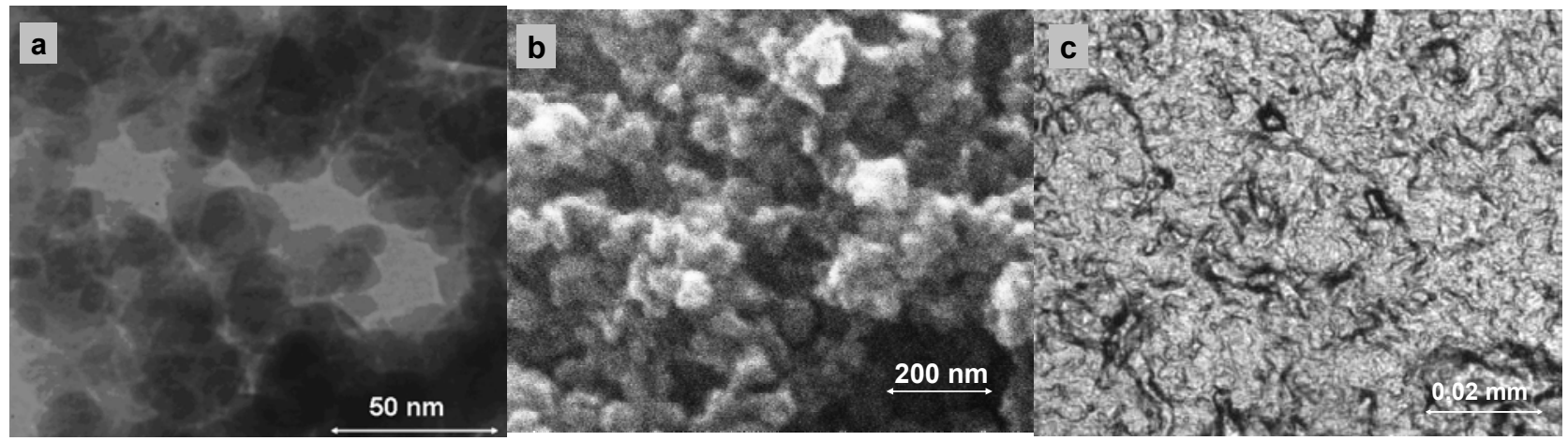

Figure 1. TEM (a), and SEM (b) microphotographs of $\mathrm{Fe}_{3} \mathrm{O}_{4} / \mathrm{PANI}$ nanocomposite powders and surface structure of $\mathrm{Fe}_{3} \mathrm{O}_{4} / \mathrm{PANI}$ thin film (c) observed by optical microscopy. 
Figure 1 shows the images of the composite film taken by different techniques at different scales. TEM images and microdiffraction patterns of the iron oxide nanoparticles of $\mathrm{Fe}_{3} \mathrm{O}_{4} / \mathrm{PANI}(1: 0.2)$ composite confirm the presence of cubic cell $\mathrm{Fd} 3 \mathrm{~m}$ nanoparticles of average size of $20 \mathrm{~nm}$. At the same time certain distribution of the size of nanoparticles was observed in the range of 10 to $40 \mathrm{~nm}$. The polymer matrix prevents agglomeration of the iron oxide and hence small separate clusters are observed being covered by polymer film, which in many cases appears to be amorphous. Due to specific contrast near the surface of the particle one can suppose that they do not have a direct contact with each other because of the shell of the polymer covering.

Magnetic measurements (Figures 2 and 3) evidence a ferromagnetic behaviour at room temperature of the obtained composite with saturation magnetization of about $3.4 \mathrm{emu} / \mathrm{g}$ and coercivity of about 40 Oe. Although the average size of the nanoparticles of the composite is close to the corresponding limit for the particles to show the superparamagnetic behaviour no contribution was observed which can be described as superparamagnetic for room temperature in frame of the accuracy of the employed experimental methods. Figure 3 shows ZFC-FC curves of the film. It is seen that there is no well-defined peak in the ZFC curve, which corresponds to the mean blocking temperature of SPM cluster.

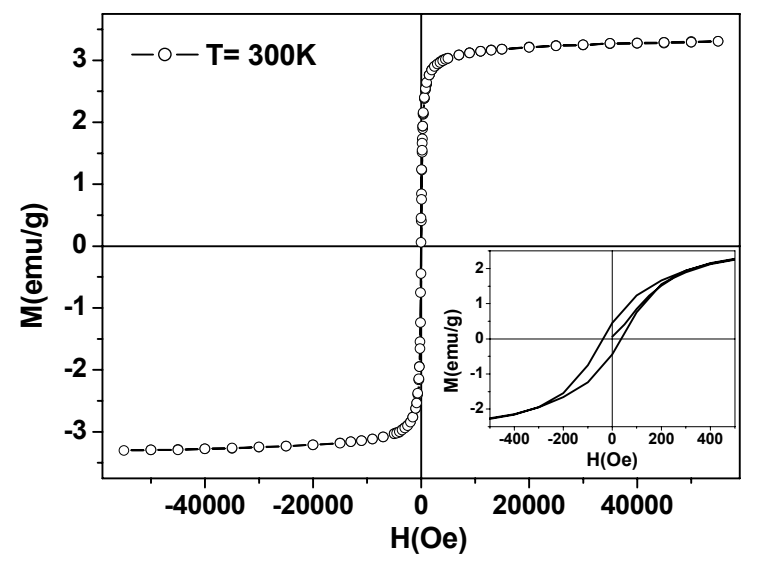

Figure 2. $\mathrm{M}$ vs $\mathrm{H}$ curve with primary magnetization curve measured in plane of $\mathrm{Fe}_{3} \mathrm{O}_{4} / \mathrm{PANI}$ thin film composite.

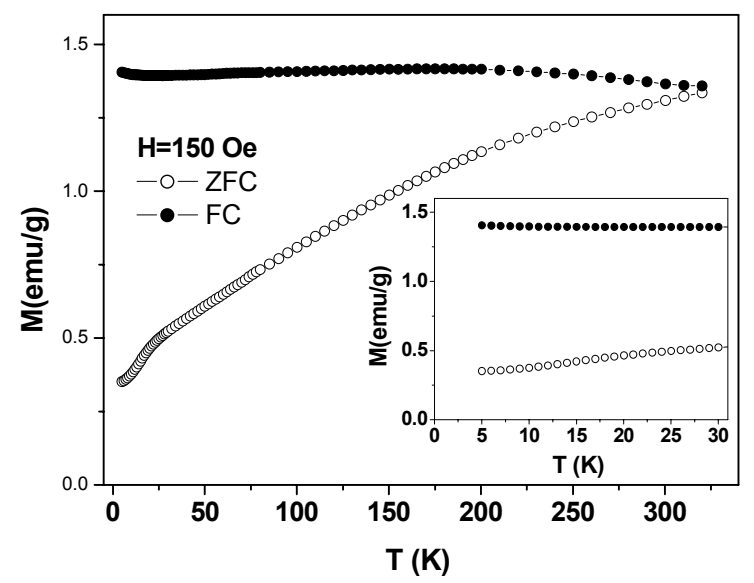

Figure 3. ZFC-FC behaviour of $\mathrm{Fe}_{3} \mathrm{O}_{4} / \mathrm{PANI}$ thin film composite.

The sample in the present study exhibit lower conductivity $(\sim 50 \mathrm{~S} / \mathrm{cm})$ than that observed in other studies, although the conductivity of the nanocomposite films decreases with increasing $\mathrm{Fe}_{3} \mathrm{O}_{4}$ [7]. Several parameters like doping level, time of drying and the medium of preparation have 
controlling influence on the conductivity of the polyaniline samples. Temperature dependence of conductivity of the composite film without (F1) and in the presence of a magnetic field (1T) (F2) exhibits a typical semiconductor behavior as it is shown in Figure 4. The inset clear indicates that for F1, the dependence of $\sigma(\mathrm{T})$ can be divided in three regions $(300-253 \mathrm{~K} ; 253-130 \mathrm{~K}$; $130-20 \mathrm{~K})$. Over a limited temperature range below room temperature, $\sigma(\mathrm{T})$ has a positive temperature coefficient (TCR). The positive TCR extends down to $250-253 \mathrm{~K}$, the conductivity increases by more than $5 \%$ of room temperature value between $300 \mathrm{~K}$ and the maximum at $253 \mathrm{~K}$ (shown in the inset to Fig.4). The positive TCR, which is generally considered arising from the carriers' scattering along the chains by phonons in metallic regions [10,11], indicates the quasi-1D nature of conduction along polymer chains [12]. It is supposed that carriers will be scattered only back along the chains, which requires phonons of relatively high energy [13]. Similar results have been reported by J.Li et al, for PANI films [14]. Both samples profiles (F1 and F2) have a strongly temperature dependent conductivity over the entire temperature interval from 253 down to $130 \mathrm{~K}$ (F1) and 300 down to $150 \mathrm{~K}$ (F2). In these ranges the film exhibits a typical semiconductor behavior, and it can be expressed by the one dimensional variable range hopping (1D-VRH) model proposed by Mott [15] as follows:

$\sigma(T)=\sigma_{o} \exp \left[-\left(\frac{T_{o}}{T}\right)^{1 / 2}\right]$

$T_{o}=8 \alpha / Z N\left(E_{F}\right) K_{B}$

where $\alpha^{-1}$ is the location length, $N\left(E_{F}\right)$ the density of states at the Fermi level, $K_{B}$ the Boltzman constant, and $\mathrm{Z}$ the number of nearest neighbor chains. Thus, plotting $\ln \sigma$ vs. $T^{1 / 2}$ as shown in Figure 4 one can obtain the $T_{o}$ value corresponding to the effective energy separation between localized states which is a measure of the degree of disorder in amorphous region. These plots exhibit a well linear dependence having a linearity factor of 0.9784 and 0.9760 . As shown in figure 4 the characteristic Mott temperature $T_{o}$ increases from $1.510^{3} \mathrm{~K}(\mathrm{~F} 1)$ to $7.910^{3} \mathrm{~K}(\mathrm{~F} 2)$ for the sample measured without and with a magnetic field (1T), respectively. Application of 1T magnetic field increases the resistivity and the slope of the $\ln \sigma$ vs $\mathrm{T}^{-1 / 2}$ dependence in F2. The positive magnetoresistance of PANI films was reported A.K.Mukherjee et al [16]. On the other hand, F1 exhibit that the linear dependence of $\ln \sigma$ on. $T^{1 / 4}(0.9816)$ is better than that of $\ln \sigma$ on. $T^{1 / 2}$ (Figure not shown).It seems to indicate that both 1D-VRH and 3D-VRH models are suitable for the conductive mechanism in F1. 


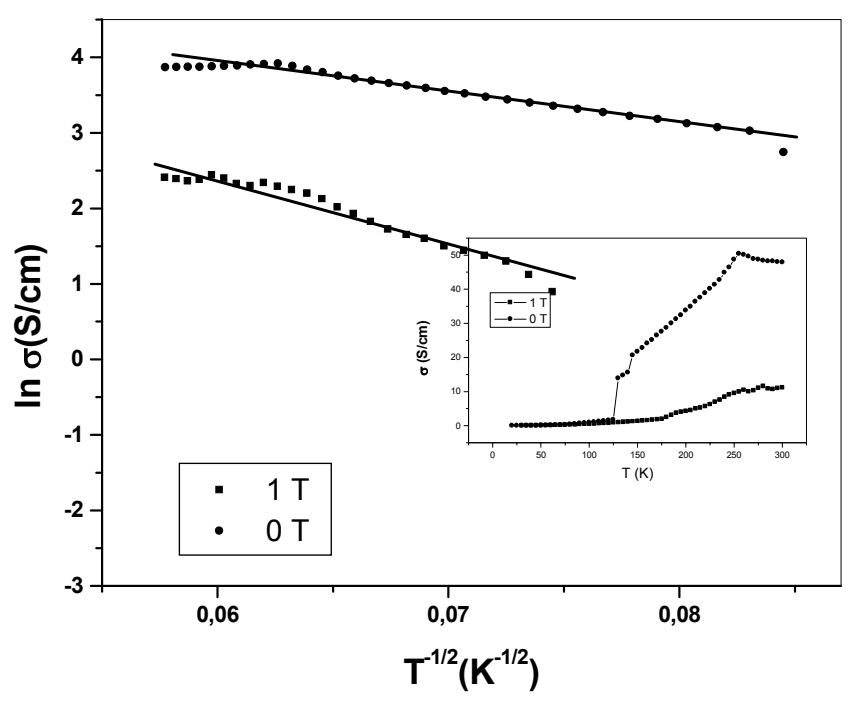

Figure 4. Temperature dependence of the conductivity of the film as prepared (F1) and in the presence of a magnetic field (F2) plotted as $\ln \sigma$ vs. $\mathrm{T}^{-1 / 2}$. Inset: temperature dependence of conductivity for both samples

\section{Conclusions}

$\mathrm{Fe}_{3} \mathrm{O}_{4} / \mathrm{PANI}$ nanoparticles of average size of $20 \mathrm{~nm}$ have been synthesized to prepare thin films with both electrical and magnetic properties. The polymer matrix prevents agglomeration of the iron oxide and hence small separate clusters are observed. The obtained composite shows a ferromagnetic behaviour at room temperature with saturation magnetization of about $3.4 \mathrm{emu} / \mathrm{g}$ and coercivity of about 40 Oe. Over a limited temperature range below room temperature, $\sigma(\mathrm{T})$ has a positive temperature coefficient (TCR). Application of a magnetic field increases the resistivity and the slope of the $\ln \sigma$ vs $\mathrm{T}^{-1 / 2}$ dependence in F2. Temperature dependence of conductivity of the composite film without (F1) and in the presence of a magnetic field (1T) (F2) exhibits a typical semiconductor behavior.

\section{Acknowledgements}

We thank N.N. Schegoleva (Institute of Metal Physics UD RAS) for the assistance with TEM study. This work was supported by the University of Buenos Aires (grant I-055). G.V. Kurlyandskaya acknowledges University of the Basque Country UPV-EHU and Spanish MEC for Ramon y Cajal Research Fellowship. Magnetic measurements and SEM observations were done at common services of the UPV-EHU.

\section{References}

1. J.L.Dormann, D.Fiorani, Magnetic Properties of Fine Particles, Eds: North-Holland, Amsterdam, 1992.

2. P.Moriarty, Rep.Prog.Phys. 64297 (2001)

3. P.Sharma, A.Gupta, K.V.Rao, F.J.Owens, R.Ahuja, J.M.O.Guillen, B.Johansson and G.A.Gehring, Nature Materals, 2673 (2003)

4. G.V. Kurlyandskaya, V.I. Levit, Biosensors \& Bioelectronics, 201611 (2005). 
5. E. Mayes, A. Bewick, D. Gleenson, J. Hoinville, R. Jones, O. Kasyutich, A. Nartowski, B. Warne, J. Wiggins, K.K.W. Wong, IEEE Trans. Magn. 39 (2) 624 (2003).

6. M.Wan, W.Li: J.Polym.Sci.Polym.Chem. 35 (1997), p.2129

7. B.Z.Tang, Y.Geng, J.W.Y.Lam and B.Li: Chem.Mater. 11, (1999), p.1581

8. Aphesteguy, J., Jacobo, S., Physica B 354 (2004) 224

9. Stejskal, J., Sapurina, I., Macromolecules 31 (1998) 2218

10. A.B.Kaiser, C.J.Liu, P.W.Gilberg, P.Chapman, N.T.Kemp, B.Wesling, A.C.Partridge, W.T.Smith, J.S.Shapiro: Synth.Met.84 (1997), p.699

11. A.B.Kaiser: Rep.Prog.Phys. 64 (2001), p.1

12. W.Li, M.Wan: Synth.Met. 92, (1998), p.121

13. P.Pipinys, A.Kiveris:Physica B 370 (2005) p.168

14. J.Li, K.Fang, H.Qiu, Sh.Li, W.Mao: Synth.Met. 142, (2004), p.107

15. N.F.Mott and E.A.Davis: Electronic Processis in Non-Crystalline Materials (1979), Oxford: Oxford University Press.

16. A.K.Mukherjee and R.Menon: J.Phys: Condens.Matter 17 (2005) p.1947 